\title{
A sobering addition to the literature on COVID-19 and the brain
}

\author{
Amit Mahajan ${ }^{1}$ and Graeme F. Mason ${ }^{1,2}$ \\ 'Department of Radiology \& Biomedical Imaging and 'Departments of Psychiatry and Biomedical Engineering, Yale University, School of Medicine, New Haven, Connecticut, USA.
}

\begin{abstract}
Several coronavirus disease 2019 (COVID-19) studies have focused on neuropathology. In this issue of the $J C I$, Qin, Wu, and Chen et al. focused specifically on people whose acute infection lacked obvious neurological involvement. Severely infected patients showed abnormal gray matter volumes, white matter diffusion, and cerebral blood flow compared with healthy controls and those with mild infection. The data remain associative rather than mechanistic, but correlations with systemic immune markers suggest effects of inflammation, hypercoagulation, or other aspects of disease severity. Mechanistic research is warranted. Given the lack of obvious neurological symptoms, neurocognitive assessments were not performed, but the findings suggest that such assessments may be warranted in severely affected patients, even without obvious symptoms. Further, studying CNS involvement of other disorders with overlapping pathophysiologies such as inflammation, coagulation, hypoxia, or direct viral infection may reveal the causes for COVID-19-related neuropathology.
\end{abstract}

\section{The absence of obvious} neurological symptoms

We read with interest the article in this issue of the JCI by Qin, $\mathrm{Wu}$, and Chen et al. (1). Patients with severe coronavirus disease 2019 (COVID-19) infection without neurological symptoms, assessed three months after infection, showed cortical thinning, reduced cerebral blood flow, and microstructural alterations in white matter. The authors saw widespread decreases in volume, length, and mean fractional anisotropy in association, commissural, projection, and limbic fiber bundles in the diseased patients when compared with healthy controls. The results are generally consistent with recent reports of CNS effects of COVID-19, but what sets this study apart is the substantial impact of severe illness even in the absence of obvious neurological symptoms.

\section{Comparing studies}

Numerous brain imaging studies of COVID-19 are emerging, and we compare two. One study included only those patients with severe illness and severely affected neurological systems, with reports of microhemorrhagic lesions in the majority of the patients, including in the white matter and especially in the medial temporal lobe (2). In the second study, which covered 60 subjects, $68 \%$ of the patients had acute neurological symptoms, and when imaged approximately three months later, $55 \%$ still had symptoms (3). Patients with COVID-19 had higher bilateral gray matter volumes in olfactory cortices, hippocampi, insulae, left Rolandic operculum, left Heschl's gyrus, and right cingulate gyrus, accompanied by an increase of fractional anisotropy in white matter, compared with volunteers who did not have COVID-19.

\section{Related Article: https://doi.org/10.1172/JCl147329}

Conflict of interest: GFM is an author on US patent application 10,770,276, titled "Techniques of mass spectrometry for isotopomer analysis and related systems and methods."

Qin, $\mathrm{Wu}, \mathrm{Chen}$, and colleagues propose that the findings likely arise from indirect injury due to hypoxia or inflammatory storm induced by the immune response (1). A neuropathological study of brain specimens from patients who died 0 to 32 days after onset of COVID-19 symptoms showed hypoxic changes without evidence of encephalitis or other specific brain changes referable to direct action of the virus (4). In another autopsy study, endothelial cell damage in the brain and a hypercoagulable state leading to neuronal hypoxia were believed to cause the neuropsychiatric symptoms/delirium seen in these patients (5).

As usual, advances lead to further questions. Qin, $\mathrm{Wu}$, and Chen et al. (1) report CNS involvement in the absence of obvious CNS symptoms, which is a finding that highlights our need to uncover mechanisms of CNS harm by COVID-19, including the closely related question of how specific the observations are for this virus. A variety of infectious conditions, including infections by severe acute respiratory syndrome-COVID (SARS-COVID) and Middle East respiratory syndrome-COVID (MERS-COVID) (6), also can damage the brain, and that fact may help guide the search for causes. Volumetric losses, in some cases with decreased diffusion anisotropy, have been reported with HIV alone (7) and comorbid with hepatitis C (8), and even with systemic inflammation with no apparent infection (9).

Sepsis can be considered a welldescribed example of brain involvement in an inflammatory syndrome, manifest by early and acute encephalopathy, seen in $70 \%$ of patients in one report (10). Patients with sepsis may present with fluctuating mental status changes, inattention, or disorganized thinking (delirium), which is associated with increased morbidity and mortality (11). Although older age, severity of illness, and metabolic disturbances are well-defined risk factors, long-term sequelae are also well 
described. Longer duration of delirium is associated with decreased brain volume as long as three months after discharge, and with long-term cognitive impairment up to 12 months (12). Duration of delirium in the intensive care unit (ICU) is also associated with white matter disruption at both discharge and three months later and with worse cognitive scores up to a year later (13). A systematic review showed that common bacterial infections are likely related to increased risk of subsequent dementia, although the overall quality of evidence was rated low (14).

Reduction of hippocampal volume, especially in the presubiculum, has been described in patients with sepsis and is negatively correlated with Acute Physiology and Chronic Health Evaluation II scores (15). Permanent cognitive impairment was demonstrated in several domains in both septic and nonseptic ICU survivors. Sepsis survivors showed cognitive deficits in verbal learning and memory with a reduction of left hippocampal volume compared with healthy controls (16). Sepsis induces degradation of the endothelial glycocalyx, mediated by heparanase, releasing highly sulfated domains of heparan sulfate into the circulation. These highly sulfated domains selectively target the hippocampus, sequestering brain-derived neurotrophic factor there, which may impact spatial memory (17).

\section{The question of inflammation}

The authors investigated the contribution of inflammation by comparing inflammatory markers with the imaging observations. Statistical significance was seen only in those with severe infection. One must keep in mind that the large number of measured parameters, relative to the necessarily exploratory sample size, opens the possibility for false positives. However, exploration is appropriate at this early stage of understanding the impact of COVID-19. Generally, greater systemic inflammatory markers were associated with greater deviation from normal in the patients with severe disease (1). Although the observational nature of Qin, Wu, and Chen et al. (1) confines the findings to associative relationships, the correlations suggest that the pursuit of more mechanistic investigations of the relationships are worthwhile. It will be important to distinguish among intertwined mechanisms, including hypercoagulation and inflammation (18).

Future work along the lines explored by Qin, Wu, and Chen et al. (1) would benefit from greater information about follow-up in individual patients, as well as a thorough neuropsychological battery to reveal potential deficits that may avert detection on a routine workup. Thorough neurocognitive and psychiatric assessments may inform us about the relationships of neuroimaging measures, functional consequences, and their trajectories.

The use of automated analysis routines, such as the ones these authors used, is desirable to minimize the possibility of bias. However, assessments of measurement reproducibility may reveal how much variability arises from the acquisition and analysis methods relative to the biological variations from day to day or between subjects. If researchers continue to pursue such approaches across sites to increase sample sizes, standardizing procedures as well as assessing neuropsychiatric and cognitive status at follow-up are warranted.

\section{Conclusions}

In summary, Qin, Wu, and Chen et al. provided a rich data set that comes with a warning that neurological deficits may appear even in patients with COVID-19 who were not seen to have CNS involvement (1). To understand the causes behind this and other reports, it will be necessary to apply similar techniques to study other disorders whose pathophysiologies, such as inflammation, coagulation, hypoxia, or direct viral infection, overlap with those of COVID-19.

\section{Acknowledgments}

$\mathrm{AM}$ and GFM acknowledge support from $\mathrm{NIH}$ grants R01 DK108283 and R21 AA028628.

Address correspondence to: Graeme F. Mason, Yale University, School of Medicine, 300 Cedar Street, PO Box 208043, New Haven, Connecticut 06520, USA. Phone: 203.737.1478; Email: graeme. mason@yale.edu.

1. Qin Y, et al. Long-term changes of microstructure and cerebral blood flow in patients recovered from COVID-19 without any specific neurological manifestations. JClin Invest. 2021;e147329.

2. Kremer S, et al. Brain MRI findings in severe COVID-19: a retrospective observational study. Radiology. 2020;297(2):E242-E251.

3. Lu Y, et al. Cerebral micro-structural changes in COVID-19 patients - an MRI-based 3-month follow-up study. EClinicalMedicine. 2020;25:100484.

4. Solomon IH, et al. Neuropathological features of Covid-19. N Engl J Med. 2020;383(10):989-992.

5. Bryce C, et al. Pathophysiology of SARSCoV-2: targeting of endothelial cells renders a complex disease with thrombotic microangiopathy and aberrant immune response. The Mount Sinai COVID-19 autopsy experience [preprint]. https://doi. org/10.1101/2020.05.18.2009996. Posted on medRxiv May 22, 2020.

6. Wu Y, et al. Nervous system involvement after infection with COVID-19 and other coronaviruses. Brain Behav Immun. 2020;87:18-22.

7. Jernigan TL, et al. Effects of methamphetamine dependence and HIV infection on cerebral morphology. Am J Psychiatry. 2005;162(8):1461-1472.

8. Zahr NM. The aging brain with HIV infection: effects of alcoholism or hepatitis $\mathrm{C}$ comorbidity. Front Aging Neurosci. 2018;10:56.

9. Marsland AL, et al. Brain morphology links systemic inflammation to cognitive function in midlife adults. Brain Behav Immun. 2015;48:195-204.

10. Young GB, et al. The electroencephalogram in sepsis-associated encephalopathy. JClin Neurophysiol.1992;9(1):145-152.

11. Sonneville R, et al. Understanding brain dysfunction in sepsis. Ann Intensive Care. 2013;3(1):15.

12. Gunther ML, et al. The association between brain volumes, delirium duration, and cognitive outcomes in intensive care unit survivors: the VISIONS cohort magnetic resonance imaging study*. Crit Care Med. 2012;40(7):2022-2032.

13. Morandi A, et al. The relationship between delirium duration, white matter integrity, and cognitive impairment in intensive care unit survivors as determined by diffusion tensor imaging: the VISIONS prospective cohort magnetic resonance imaging study* . Crit Care Med. 2012;40(7):2182-2189.

14. Muzambi R, et al. Common bacterial infections and risk of dementia or cognitive decline: a systematic review. J Alzheimers Dis. 2020;76(4):1609-1626.

15. Yuan M, et al. Effects of sepsis on hippocampal volume and memory function. World J Emerg Med. 2020;11(4):223-230.

16. Semmler A, et al. Persistent cognitive impairment, hippocampal atrophy and EEG changes in sepsis survivors. J Neurol Neurosurg Psychiatry. 2013;84(1):62-69.

17. Zhang X, et al. Circulating heparin oligosaccharides rapidly target the hippocampus in sepsis, potentially impacting cognitive functions. Proc Natl Acad Sci U S A. 2019;116(19):9208-9213.

18. Jose RJ, Manuel A. COVID-19 cytokine storm: the interplay between inflammation and coagulation. Lancet Respir Med. 2020;8(6):e46-e47. 